\title{
Nonlinear surface-wave excitations in the Bénard-Marangoni system
}

\author{
R. A. Kraenkel and J. G. Pereira \\ Instituto de Física Teórica, Universidade Estadual Paulista, Rua Pamplona 145, 01405 São Paulo, São Paulo, Brazil \\ M. A. Manna \\ Laboratoire de Physique Mathématique, Université des Sciences et Techniques du Languedoc, 34060 Montpellier CEDEX, France \\ (Received 28 April 1992)
}

\begin{abstract}
We examine the appearance of surface waves governed by Burgers and Korteweg-de Vries equations in a shallow viscous heated fluid. We consider waves triggered by a surface-tension variation induced by both temperature and concentration gradients. We also establish the range of parameters for which the above-mentioned equations appear.
\end{abstract}

PACS number(s): 47.20.Bp, 47.35. $+\mathrm{i}$

\section{INTRODUCTION}

It is by now a well-established fact that surface-tension gradients may drive motion in convective systems. For instance, the convective instability found in the original experiments performed by Bénard has been shown, both experimentally [1] and theoretically [2], to be of this kind. It is usually termed Bénard-Marangoni instability. It is dominant with respect to buoyancy effects (Rayleigh phenomenon) for fluids of small depth, as can intuitively be seen from the adimensional parameters relevant to the stability analysis, i.e., Marangoni and Rayleigh numbers. The first depends on the square of the undisturbed depth, while the second depends on the fourth power.

The situations we will be interested in concern fluid layers of infinite horizontal extent, bounded below by a plate, and with a free upper surface. Considering just buoyancy effects, Alfaro and Depassier [3] have shown that, when the Rayleigh number $R=30$, a long-wave instability sets in, governed by the Korteweg-de Vries $(\mathrm{KdV})$ equation. In the same context, we have shown [4] that the Burgers equation governs the perturbations of the free surface for $R \neq 30$. In this way a connection between integrable equations, such as $\mathrm{KdV}$ and Burgers, and hydrodynamical instabilities has been established.

A natural question to pose is whether surface-tension gradients may also drive such long-wave instabilities. It is this question that will be addressed here. We have first to specify what causes such surface-tension gradients. Two well-known sources are temperature and concentration gradients, and both will be taken into account. They are not completely uncorrelated, since temperature gradients induce concentration gradients, the so-called Soret effect. This effect will also be considered in our analysis. Steps in this direction have been taken by other authors [5-7] for the case of thermal gradients only, indicating that for Marangoni number $\mathbf{M a}=-12$, an instability governed by the $\mathrm{KdV}$ equation must appear. This result can be recovered as a particular case of our solutions.

\section{BASIC EQUATIONS}

We will consider a fluid layer bounded below, at $z=0$, by a perfectly conducting plate, and above by a free surface located at $z=d$ when at rest. We will be dealing with a two-dimensional system, the horizontal coordinate being denoted by $x$. The horizontal and vertical components of the velocity will be denoted respectively by $u$ and $w$. The pressure will be denoted by $p$, the temperature by $\theta$, and the concentration by $C$. The density $\rho$ will be considered constant, which means that buoyancy will be neglected.

The fundamental equations governing this system are

$$
\begin{aligned}
& u_{x}+w_{z}=0, \\
& \rho\left(u_{t}+u u_{x}+w u_{z}\right)=-p_{x}+\mu\left(u_{x x}+u_{z z}\right), \\
& \rho\left(w_{t}+u w_{x}+w w_{z}\right)=-p_{z}+\mu\left(w_{x x}+w_{z z}\right)-g \rho, \\
& \theta_{t}+u \theta_{x}+w \theta_{z}=\kappa_{T}\left(\theta_{x x}+\theta_{z z}\right), \\
& C_{t}+u C_{x}+w C_{z}=\kappa_{S}\left(C_{x x}+C_{z z}\right)+\operatorname{So}\left(\theta_{x x}+\theta_{z z}\right) .
\end{aligned}
$$

In the above equations, subscripts indicate derivation, $\mu$ is the viscosity, $\kappa_{T}$ is the coefficient of thermal diffusivity, $\kappa_{S}$ is the coefficient of solutal diffusivity, So is the Soret coefficient, and gravity has been taken into account through

$$
\mathbf{g}=-g \rho \mathbf{k} \text {. }
$$

We must now supplement those equations with appropriate boundary conditions. Let $\eta(x, t)$ denote the surface displacement in relation to the quiescent state, so that the free surface is located at $z=d+\eta(x, t)$. Then, at the surface we must have [8]

$\eta_{t}+u \eta_{x}=w$

$p-p_{a}-\frac{2 \mu}{N^{2}}\left[w_{z}+u_{x}\left(\eta_{x}\right)^{2}-\eta_{x}\left(u_{z}+w_{x}\right)\right]=-\frac{T}{N^{3}} \eta_{x x}$ 


$$
\begin{aligned}
& \mu\left[1-\left(\eta_{x}\right)^{2}\right]\left(u_{z}+w_{x}\right)+2 \mu \eta_{x}\left(w_{z}-u_{x}\right) \\
& =N\left(T_{x}+\eta_{x} T_{z}\right), \\
& -\eta_{x} \theta_{x}+\theta_{z}=-\frac{F}{k_{T}} N \\
& -\eta_{x} C_{x}+C_{z}=-\frac{J}{k_{S}} N
\end{aligned}
$$

We have denoted by $p_{a}$ the constant pressure exerted on the upper surface, by $T$ the surface tension, and by $k_{T}$ and $k_{S}$, the thermal and solutal conductivities, respectively. The fluxes normal to the free surface, indicated by $F$ and $J$, were assumed to be constant. Finally, $N$ is defined by $N=\left[1+\left(\eta_{x}\right)^{2}\right]^{1 / 2}$. On the lower plate we will impose stress-free boundary conditions,

$$
w=u_{z}=0,
$$

and that the temperature and concentration be constant:

$$
\theta(x, 0, t)=\theta_{b}, \quad C(x, 0, t)=C_{b} .
$$

The surface tension depends on temperature and concentration in the following form:

$$
T=T_{r}\left[1-\gamma\left(\theta-\theta_{r}\right)-\beta\left(C-C_{r}\right)\right],
$$

where $T_{r}, \theta_{r}, C_{r}$ are reference values, and $\gamma, \beta$ are constants.

We now adimensionalize all quantities by choosing $d$ as the unit of length, $\rho d^{3}$ as the unit of mass, $F d / k_{T}$ as the unit of temperature, $J d / k_{S}$ as the unit of concentration, and $(d / g)^{1 / 2}$ as the unit of time. In convection phenomena, $d^{2} / k_{T}$ is sometimes used as the time scale, but we prefer not to use it here in order to maintain the symmetry between thermal and solutal quantities. In this case, the following adimensional numbers will appear in the equations: the Galileo number $G=\rho^{2} g d^{3} / \mu^{2}$; the Prandtl number $\sigma=\mu / \rho \kappa_{T}$; the Schmidt or solutal Prandtl number $\sigma_{S}=\mu / \rho \kappa_{S}$; the Marangoni number $\mathrm{Ma}$ $=F d^{2} T_{r} \gamma / k_{T} \kappa_{T} \mu$; the solutal Marangoni number $\mathrm{Mn}=J d^{2} T_{r} \beta / k_{S} \kappa_{S} \mu$; the Bond number $B=g \rho d^{2} / T_{r}$; and a new number, which we will call the Soret number So $=\beta S / \gamma \kappa_{S}$.

In order to perform perturbation theory, we have to introduce a reference state from which small departures will be considered. This state will be the static one, given by $u=w=0$, and

$$
\begin{aligned}
& \theta_{s}=\theta_{r}-(z-1), \\
& C_{s}=C_{r}-(z-1), \\
& P_{s}=P_{a}-(z-1), \\
& T_{s}=\frac{1}{B}+\frac{\mathrm{Ma}}{G \sigma}(z-1)+\frac{\mathrm{Mn}}{G \sigma_{s}}(z-1) .
\end{aligned}
$$

These relations are already in an adimensional form. Since we are interested in long-wavelength perturbations, we will use the reductive perturbation method of Taniuti [8]. It consists basically in introducing a small parameter $\epsilon$, and defining stretched coordinates through

$$
\xi=\epsilon(x-a t), \quad \tau=\epsilon^{\delta} t,
$$

where $a$ is a yet undetermined constant, and $\delta$ will be either 2 , if we want to look for dissipative effects, or 3 , if we want to look for dispersive effects. All dependent variables will then be written as perturbative series in $\epsilon$, and an order-by-order solution to the system of equations will be found. This is what will be done in the next sections.

\section{THE DISSIPATIVE CASE}

Once the adimensional variables and stretched coordinates (with $\delta=2$ ) have been introduced, we proceed further by introducing the following expansions:

$$
\begin{aligned}
& u=\epsilon\left(u_{0}+\epsilon u_{1}+\epsilon^{2} u_{2}+\cdots\right), \\
& w=\epsilon^{2}\left(w_{0}+\epsilon w_{1}+\epsilon^{2} w_{2}+\cdots\right), \\
& \eta=\epsilon\left(\eta_{0}+\epsilon \eta_{1}+\epsilon^{2} \eta_{2}+\cdots\right), \\
& \theta-\theta_{s}=\epsilon^{2}\left(\theta_{0}+\epsilon \theta_{1}+\epsilon^{2} \theta_{2}+\cdots\right), \\
& C-C_{s}=\epsilon^{2}\left(C_{0}+\epsilon C_{1}+\epsilon^{2} C_{2}+\cdots\right), \\
& p-p_{s}=\epsilon\left(p_{0}+\epsilon p_{1}+\epsilon^{2} p_{2}+\cdots\right) .
\end{aligned}
$$

With these expansions, Eqs. (1)-(11) can then be solved order by order in $\epsilon$. For the sake of brevity, however, we will not give here the calculations in full detail, since they are straightforward. Instead, we will only state the solutions for each order in $\epsilon$. The lowest order gives

$$
\begin{aligned}
& u_{0}=f(\xi, \tau), \quad w_{0}=-f_{\xi}(\xi, \tau) z \\
& p_{0}=\eta_{0}=\frac{1}{a} f(\xi, \tau), \quad \theta_{0}=\sigma \sqrt{G}\left[\frac{z^{3}}{6}-\frac{z}{2}\right] f_{\xi}(\xi, \tau), \\
& C_{0}=\sigma_{s} \sqrt{G}\left[1-\frac{\text { Ma So }}{\mathrm{Mn}}\right]\left[\frac{z^{3}}{6}-\frac{z}{2}\right] f_{\xi}(\xi, \tau),
\end{aligned}
$$

where $f(\xi, \tau)$ is an arbitrary function. In the next order we get

$$
\begin{aligned}
& u_{1}=\frac{z^{2}}{2 a \sqrt{G}}\left[\frac{\mathrm{Ma}}{\sigma}+\frac{\mathrm{Mn}}{\sigma_{s}}\right] f_{\xi}+h(\xi, \tau) \\
& w_{1}=-\frac{z^{3}}{6 a \sqrt{G}}\left[\frac{\mathrm{Ma}}{\sigma}+\frac{\mathrm{Mn}}{\sigma_{s}}\right] f_{\xi \xi}-z h_{\xi}(\xi, \tau) \\
& p_{1}=\eta_{1}-\frac{2}{\sqrt{G}} f_{\xi} \\
& a \eta_{1 \xi}-h_{\xi}=\frac{1}{a} f_{\tau}+\frac{2}{a} f f_{\xi}+\frac{1}{6 a \sqrt{G}}\left[\frac{\mathrm{Ma}}{\sigma}+\frac{\mathrm{Mn}}{\sigma_{s}}\right] f_{\xi \xi}
\end{aligned}
$$

where $h(\xi, \tau)$ is another arbitrary function. At this order, there appears a compatibility condition yielding

$$
a^{2}=1-\frac{1}{G}\left[\frac{\mathrm{Ma}}{\sigma}+\frac{\mathrm{Mn}}{\sigma_{s}}\right] .
$$

We did not calculate $T_{1}$ and $C_{1}$, as they will not be neces- 
sary in the next steps. Note that we could not write $p_{1}$ and $\eta_{1}$ explicitly in terms of $h(\xi, \tau)$. Instead, we have a functional relation between them.

Much in the same way, the next order will again yield a compatibility condition, which, this time, will involve $f(\xi, \tau)$. Indeed, it gives us an equation for $f(\xi, \tau)$, which, once solved, provides a solution to the whole lowest order. To obtain it, we have just to use Eq. (2) to calculate $\left(u_{2}\right)_{z}$ in terms of $f, h$, and $\eta_{1}$, which can be easily done. Equation (8) is a boundary condition for $\left(u_{2}\right)_{z}$ at $z=1$. Actually, it is a relation involving the functions $f, h$, and $\eta_{1}$, in which $\eta_{1}$ and $h$ enter through the combination $\left[a\left(\eta_{1}\right)_{\xi}-h_{\xi}\right]$. By using this relation in Eq. (19), we obtain an evolution equation for $f(\xi, \tau)$ :

$$
f_{\tau}+\left[1+\frac{1}{2 a^{2}}\right] f f_{\xi}=v f_{\xi \xi},
$$

where

$$
v=\frac{1}{\sqrt{G}}\left[2-\frac{\mathrm{Ma}(\mathrm{So}-1)-\mathrm{Mn}}{6}\right] .
$$

This is the Burgers equation. Through a nonlinear Hopf-Cole transformation it can be reduced to the linear heat equation and, therefore, be solved [9]. An interesting solution is obtained when we take as the initial condition a surface perturbation defined by a step function. In this case, the asymptotic $(\tau \rightarrow \infty)$ form of the solution will be the Taylor shock profile, given by

$$
f(\xi, \tau)=\lambda\left[1-\tanh \left[\frac{\lambda}{2 v}(\xi-\lambda \tau)\right],\right.
$$

where $\lambda$ is a constant. The Burgers equation also presents $N$-Taylor shock solutions, as well as rational solutions.

\section{THE DISPERSIVE CASE}

The same procedure employed in the last section will be reproduced here, but with $\delta=3$ instead of 2 . The corresponding appropriate scaling of the dependent variables are

$$
\begin{aligned}
& u=\epsilon^{2}\left(u_{0}+\epsilon u_{1}+\epsilon^{2} u_{2}+\cdots\right), \\
& w=\epsilon^{3}\left(w_{0}+\epsilon w_{1}+\epsilon^{2} w_{2}+\cdots\right), \\
& \eta=\epsilon^{2}\left(\eta_{0}+\epsilon \eta_{1}+\epsilon^{2} \eta_{2}+\cdots\right), \\
& \theta-\theta_{s}=\epsilon^{3}\left(\theta_{0}+\epsilon \theta_{1}+\epsilon^{2} \theta_{2}+\cdots\right), \\
& C-C_{s}=\epsilon^{3}\left(C_{0}+\epsilon C_{1}+\epsilon^{2} C_{2}+\cdots\right), \\
& p-p_{s}=\epsilon^{2}\left(p_{0}+\epsilon p_{1}+\epsilon^{2} p_{2}+\cdots\right) .
\end{aligned}
$$

In the lowest order, the results are

$$
\begin{aligned}
& u_{0}=f(\xi, \tau), \quad w_{0}=-z f(\xi, \tau), \\
& p_{0}=\eta_{0}=\frac{1}{a} f(\xi, \tau), \\
& T_{0}=\sigma \sqrt{G}\left[\frac{z^{3}}{6}-\frac{z}{2}\right] f_{\xi}, \\
& C_{0}=\sigma_{s} \sqrt{G}\left[1-\frac{\mathrm{Ma}}{\mathrm{Mn}} \mathrm{So}\right]\left[\frac{z^{3}}{6}-\frac{z}{2}\right] f_{\xi},
\end{aligned}
$$

with $f(\xi, \tau)$ an arbitrary function. The next order gives

$$
\begin{aligned}
u_{1}= & \frac{z^{2} \sqrt{G}}{2 a}\left[1-a^{2}\right] f_{\xi}+g(\xi, \tau), \\
w_{1}= & -\frac{z^{3} \sqrt{G}}{6 a}\left[1-a^{2} f_{\xi \xi}-z g_{\xi},\right. \\
\eta_{1}= & \frac{\sqrt{G}}{6 a^{2}}\left[1-a^{2}\right] f_{\xi}+\frac{1}{a} g, \\
p_{1}= & \frac{\sqrt{G}}{6 a^{2}}\left[1-a^{2}\right] f_{\xi}+\frac{1}{a} g-\frac{2}{\sqrt{G}} f_{\xi}, \\
T_{1}= & \frac{\sigma G}{a}\left[1-a^{2}\right]\left[\frac{z^{5}}{120}-\frac{z}{24}\right] f_{\xi \xi} \\
& +\sigma \sqrt{G}\left[\frac{z^{3}}{6}-\frac{z}{2}\right] g_{\xi}-a^{2} \sigma^{2} G \\
& \times\left[\frac{z^{5}}{120}-\frac{z^{3}}{12}+\frac{5 z}{24}\right] f_{\xi \xi},
\end{aligned}
$$

$$
\begin{aligned}
C_{1}= & \frac{\sigma_{s} G}{a}\left[1-a^{2}\right]\left[1-\frac{\mathrm{Ma}}{\mathrm{Mn}} \text { So }\right]\left[\frac{z^{5}}{120}-\frac{z}{24}\right] f_{\xi \xi}+\sigma_{s} \sqrt{G}\left[1-\frac{\mathrm{Ma}}{\mathrm{Mn}} \text { So }\right]\left[\frac{z^{3}}{6}-\frac{z}{2}\right] g_{\xi} \\
& -\left[\frac{\sigma_{s}^{2} G}{a}\left[1-\frac{\mathrm{Ma}}{\mathrm{Mn}} \mathrm{So}\right]-\frac{a \sigma_{s} \sigma G \mathrm{Ma} \text { So }}{\mathrm{Mn}}\right]\left[\frac{z^{5}}{120}-\frac{z^{3}}{12}+\frac{5 z}{24}\right] f_{\xi \xi},
\end{aligned}
$$

$$
a^{2}=1-\frac{1}{G}\left[\frac{\mathrm{Ma}}{\sigma}+\frac{\mathrm{Mn}}{\sigma_{s}}\right],
$$

compatibility condition that will lead to the vanishing of the coefficient of the dissipative term:

$$
\mathrm{Ma}(1-\mathrm{So})+\mathrm{Mn}=-12 \text {. }
$$

This is exactly what should be expected from the coherence of the reductive perturbation method. We will not write out the full expressions for $u_{2}, w_{2}, \ldots$, as they are quite involved. A further step must be given in the per-
In contrast to the dissipative case, the next order does

not give us an equation for $f(\xi, \tau)$. Instead, it gives us a where again $g(\xi, \tau)$ is an arbitrary function. Equation (32) is the compatibility condition appearing at this order. 
turbative expansions in order to find the equation governing $f(\xi, \tau)$. It is analogous to the dissipative case: first, one calculates $u_{3 z}$ from Eq. (2) and substitute it in Eq. (8), using all the preceding results. This will lead to the equation

$$
f_{\tau}+P f f_{\xi}+Q f_{\xi \xi \xi}=0
$$

where

$$
\begin{aligned}
P= & 1+\frac{1}{2 a^{2}}, \\
Q= & \frac{7 a}{15}-\frac{3}{10 a}-\frac{1}{2 a B} \\
& -\frac{a}{15}\left[\mathrm{Ma} \sigma+\operatorname{Mn} \sigma_{s}-\operatorname{MaSo}\left(\sigma+\sigma_{s}\right)\right],
\end{aligned}
$$

with a satisfying Eq. (32). Equation (34) is the wellknown Korteweg-de Vries equation, which appears in many branches of physics when systems with weak nonlinearity and dispersion are considered. This equation has been extensively studied by several methods [10], and we know that it has rational, as well as $N$-soliton solutions. Its one-soliton solution is given by

$$
f(\xi, \tau)=\frac{12}{P} \lambda^{2} \operatorname{sech}^{2}\left[\frac{\lambda}{\sqrt{Q}}\left(\xi-4 \lambda^{2} \tau\right)\right],
$$

where $\lambda$ is a parameter characterizing $f(\xi, 0)$.

\section{DISCUSSION OF THE RESULTS}

From the above analysis we can see that if the criticality condition, Eq. (33), holds, the evolution of the free surface is governed by the $\mathrm{KdV}$ equation. Otherwise, it will be governed by the Burgers equation. It should be mentioned, however, that the transition from the Burgers to the $\mathrm{KdV}$ equation, and vice versa, is not an abrupt transition, but a smooth one. This can be seen in the following way. First we notice that, since we are using a perturbative expansion, higher-order corrections exist. For example, in the case of the Burgers equation $(\delta=2)$, the dispersive term of $\mathrm{KdV}$ equation does appear as a higher-order term, yielding actually the KdV-Burgers equation. Far away from the criticality condition, however, the higher-order dispersive term can be neglected, leading to the Burgers equation. On the other hand, near enough to the criticality condition, the coefficient of the dissipative term becomes vanishingly small, and the higher-order dispersive term becomes important. In this region, the $\mathrm{KdV}$-Burgers equation appears as the equation governing surface excitations. When the criticality condition is satisfied, the dissipative term vanishes, and by choosing the appropriate value $\delta=3$, we obtain the $\mathrm{KdV}$ equation. This smooth transition is not explicit in our approach because we have used the reductive perturbation method, which compares orders in $\epsilon$, and the dispersive and dissipative terms of the $\mathrm{KdV}$-Burgers equation appear in different orders. Moreover, since this method makes use of the stretched coordinates $\xi$ and $\tau$, the order of the dispersive and dissipative terms are $\delta$ dependent. The reader is addressed to Ref. [12] for a full discussion of these aspects.
Let us now examine the consequences of the results obtained so far. In both the $\mathrm{KdV}$ and Burgers equations, the nonlinear term is the same, and is independent of the Soret number. However, the integrability condition, Eq. (32), must be taken into account. Clearly, since $a$ is real, it must hold that

$$
\frac{\mathrm{Ma}}{\sigma}+\frac{\mathrm{Mn}}{\sigma_{s}}<G
$$

When $\mathrm{Ma} / \sigma+\mathrm{Mn} / \sigma_{s}$ approaches $G, a^{2}$ goes to zero, and the coefficient of the nonlinear term, $\left[1+(2 a)^{-2}\right]$, goes to infinity. If Eq. (37) is not satisfied, then we would have $f=0$, meaning that no long-wave instability would be present.

We discuss now the case So $=0$. The sign of the coefficient of the second derivative term in Eq. (21) is positive if $(\mathrm{Ma}+\mathrm{Mn})>-12$, and represents dissipation. In this case Eq. (21) is just the usual Burgers equation. However, if $(\mathrm{Ma}+\mathrm{Mn})<-12$, the second derivative coefficient is negative, indicating antidissipation or selfexcitation. In this range of parameters, small perturbations would grow indefinitely, but we should bear in mind that it could not be so, as higher-order terms may possibly be important in damping the instability [11]. It is also worth pointing out that in both cases a kink solution exists [4], but its stability depends again on higher-order calculations. When $(\mathrm{Ma}+\mathrm{Mn})=-12$, we have an energy balance, and the surface perturbations will be governed by the $\mathrm{KdV}$ equation.

The situation can best be visualized in a diagram on a Ma $\times$ Mn plane, as shown in Fig. 1. First of all, let us consider the most usual case where $\sigma_{s}>\sigma$. The full line represents $(\mathrm{Ma}+\mathrm{Mn})=-12$, and the dashed one represents the case where $\left(\mathrm{Ma} / \sigma+\mathrm{Mn} / \sigma_{s}\right)=G$. In region $B$, the surface perturbations are governed by the "dissipative" Burgers equation (with So $=0$ ), and in region $C$ by the "antidissipative" Burgers equation. On the line separating $B$ from $C$, the evolution equation of surface perturbations is the $\mathrm{KdV}$ equation.

The two lines in Fig. 1 intercept each other at the point

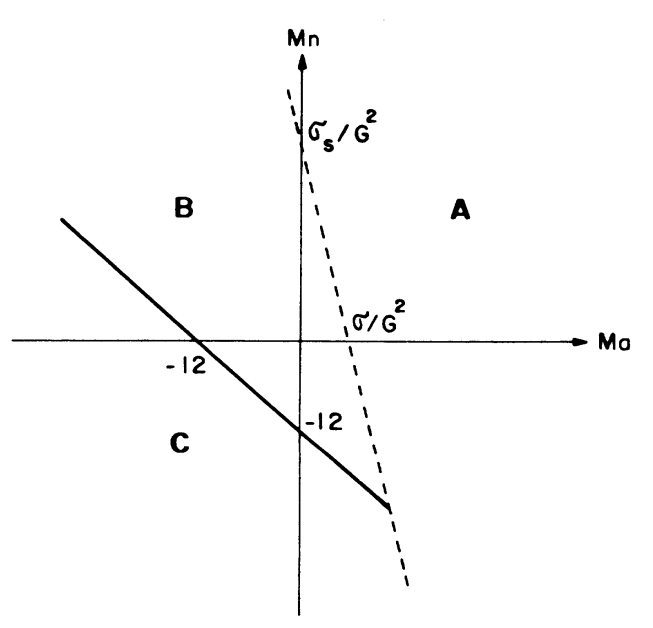

FIG. 1. Diagram on the plane Ma-Mn, showing the regions where different evolution equations appear, for the case $\mathrm{So}=0$. 


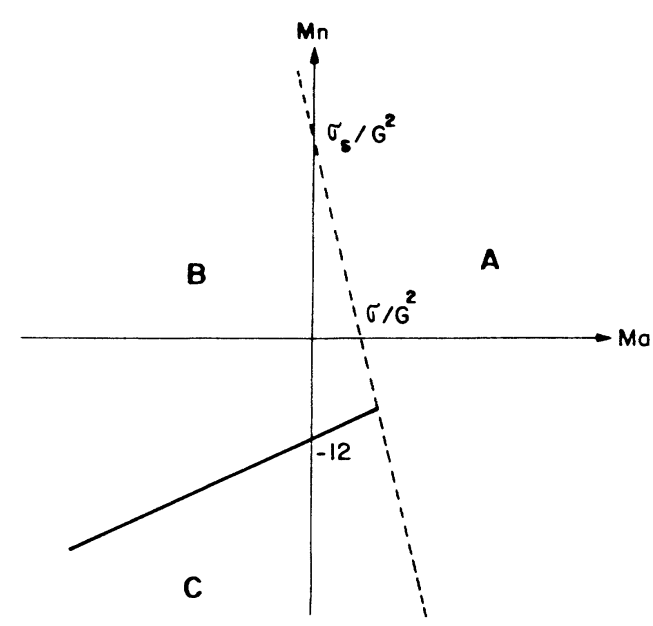

FIG. 2. Diagram on the plane Ma-Mn, showing the regions where different evolution equations appear, for the case So $>0$.

given by the coordinates

$$
\mathrm{Ma}^{*}=\frac{\left(12+\sigma_{s} G\right) \sigma}{\sigma_{s}-\sigma}, \quad \mathrm{Mn}^{*}=\frac{(12+\sigma G) \sigma_{s}}{\sigma-\sigma_{s}} .
$$

In the region $\mathrm{Ma}>\mathrm{Ma}^{*}$, or $\mathrm{Mn}<\mathrm{Mn}^{*}$, it is impossible to have the evolution governed either by the $\mathrm{KdV}$ or by the dissipative Burgers equation.

We are now in a position to discuss the Soret effect. If $\mid$ So $\mid \ll<1$, no qualitative changes appear: there is only a small departure from the initial inclination of the full line in Fig. 1. This is the case in which the Soret effect is small, and it is the most frequent. However, it is perfectly reasonable to have a big Soret number for substances with a large value of $\beta$. If So $\rightarrow 1$, the full line tends to become parallel to the Ma axis. For So $>1$, the situation is that depicted in Fig. 2, where the regions $A, B$, and $C$ still have the same meaning as before. The interception is at the point defined by the coordinates

$\mathrm{Ma}^{*}=\frac{\left(12+\sigma_{s} G\right) \sigma}{\sigma_{s}-(1-\mathrm{So}) \sigma}, \quad \mathrm{Mn}^{*}=\frac{[12+(1-\mathrm{So}) \sigma G] \sigma_{s}}{(1-\mathrm{So}) \sigma-\sigma_{s}}$.

For $\mathrm{Ma}>\mathrm{Ma}^{*}$, or $\mathrm{Mn}>\mathrm{Mn}^{*}$, the evolution will never be governed by the $\mathrm{KdV}$ equation. Yet, for $\mathrm{Ma}>\mathrm{Ma}^{*}$, we will never have the dissipative Burgers equation, and for $\mathrm{Mn}>\mathrm{Mn}^{*}$, we will never have the antidissipative Burgers as the equation governing the surface perturbations.

\section{CONCLUSIONS}

We have investigated the surface long-wavelength instability appearing in a Bénard-Marangoni system acted upon by heat and mass fluxes, in the limiting case of no buoyancy, and taking into account the dependence of the surface tension on both temperature and concentration gradients. We have found that a perturbation in the free surface is governed by the $\mathrm{KdV}$ equation when a certain criticality condition is satisfied. When it is not, the Burgers equation appears as the equation governing the surface perturbations. In this case, the coefficient of the dissipative term can be either positive or negative, indicating dissipation or self-excitation. Finally, we have also discussed the consequences of the Soret effect, and the qualitative changes it may introduce.

\section{ACKNOWLEDGMENTS}

We would like to thank Conselho Nacional de Desenvolvimento Cientifico e Tecnológico (CNPq), Brazil, for partial support. One of the authors (R.A.K.) also thanks FUNDUNESP for partial support.
[1] M. J. Block, Nature 178, 650 (1956).

[2] M. G. Velarde, in Fluid Dynamics, edited by R. Balian and J. L. Peube (Gordon and Breach, London, 1973).

[3] C. M. Alfaro and M. C. Depassier, Phys. Rev. Lett. 62, 2597 (1989).

[4] R. A. Kraenkel, J. G. Pereira, and M. A. Manna, Phys. Rev. A 45, 838 (1992).

[5] A. V. Porubov (unpublished).

[6] A. N. Garazzo and M. G. Velarde, Phys. Fluids A 3, 2295 (1991).

[7] R. D. Benguria and M. C. Depassier, Phys. Fluids A 1,
1123 (1989).

[8] T. Taniuti, Suppl. Prog. Theor. Phys. 55, 1 (1974).

[9] G. B. Whitham, Linear and Nonlinear Waves (Wiley, New York, 1974).

[10] M. J. Ablowitz and H. Segur, Solitons and the Inverse Scattering Transform, SIAM Studies in Applied Mathematics (Society for Industrial and Applied Mathematics, Philadelphia, 1981).

[11] T. Kawahara, Phys. Rev. Lett. 51, 381 (1983).

[12] G. L. Lamb, Jr., Elements of Soliton Theory (Wiley, New York, 1980). 